2005;115:1360-1366). (Respond: Alexandra J Richardson DPhil, Oxford University Laboratory of Physiology, Parks Road, Oxford OX1 3PT, UK).

COMMENT. Developmental coordination disorder (DCD) is a motor skills disorder, not due to cerebral palsy or muscular dystrophy, that impairs a child's performance in sports or handwriting and interferes with academic achievement or activities of daily living (DSMIV-R, 2000). In neurological terms, DCD is equivalent to an ideokinetic (adextrous) dyspraxia (Denny-Brown D. 1957), or clumsiness, in writing, dressing, using scissors and other ADL, despite normal muscle power (a dominant parietal lobe dysfunction), and it overlaps with the syndromes of ADHD and DAMP (deficit in attention, motor control and perception) (Landgren $\mathrm{M}$ et al, 1996). Treatment involves occupational therapy, special education, and, when indicated, medication. The above authors demonstrate a beneficial effect of fatty acid supplements in the diet in children with reading, spelling, attention and behavior disorders, but without improvement in coordination. These findings confirm eariier reports of the benefits of docosahexaenoic acid supplements in dark adaptation (scotopic vision) and improvements in reading ability in some dyslexics (Stordy BJ, 1995; Ped Neur Briefs Nov 1995). They also corroborate the report of a significant decrease in serum essential fatty acids (docosahexaenoic acid etc) found in 44 hyperactive children compared to 45 age- and sex-matched controls (Mitchell EA et al, 1987; Ped Neur Briefs Sept 1987). The hyperactive children had a greater incidence of learning disabilities and dyslexia than controls, but a double-blind, placebo-controlled, crossover study of evening primrose oil in 31 patients showed only modest improvements in behavior.

The present authors cite more recent references to successful trials of $w-3 / w-6$ fatty acid supplements in children with dyslexia or ADHD, but a lack of response to w-3 fatty acid (docosahexaenoic acid) alone. The combination of omega-3 and omega-6 (linoleic) fatty acids appears to be essential, and also the dosage is probably important, in the duplication of the authors' encouraging results. Other research has claimed that deficiencies in omega-3 fatty acids and increased intake of omega- 6 linoleic acid may explain increases in crime and aggressive behavior in young adults (Hibbeln JR et al. Lipids 2004;39:1207-13). Dietary modifications or supplements in the treatment of learning and behavior disorders of children have frequently fallen short of initial expectations after closer and more prolonged study. These have included the additive-salicylate-free, hypoallergenic, sugar-restricted, megaritamin, and mineral and trace element diets (Millichap JG, Attention Deficit Hyperactivity and Learning Disabilities. Chicago, PNB Publishers, 1998). Despite the excellent experimental design of the Oxford-Durham study, further controlled trials are indicated before advocating fish, with its attendant mercury exposure, or fatty acids as a general substitute for medication. However, the introduction of a relatively safe, and well tolerated, dietary treatment could, if successful, serve as a complementary or substitute treatment, and offset the increase in concern regarding side effects of drug therapies.

\title{
CHANGES IN CEREBRAL BLOOD FLOW WITH AGE IN ADHD
}

Changes in regional cerebral blood flow (rCBF) with age were studied by SPECT in 29 drug-naïve patients with attention deficit hyperactivity disorder (ADHD) ( 24 boys, 5 girls; age 7-13) and 12 with complex partial epilepsy as controls, at NIMH International Mental 
Health Program, Istanbul; and University of Ankara Medical School, Ankara, Turkey. The prefrontal cortex rCBF during resting conditions showed increased lateralization from right to left with age in ADHD subjects, and compared to controls, ADHD was associated with lower right prefrontal/frontal and higher parietal $\mathrm{rCBF}$. These differences were greater in subjects older than 7 years. The left hemisphere dominance in the prefrontal cortex increased significantly with age in ADHD subjects, whereas the control group showed no significant correlations between age and asymmetry of rCBF. (Oner O, Oner P, Aysev A, Kucuk O, Ibis E. Regional cerebral blood flow in children with ADHD: changes with age. Brain Dev June 2005;27:279-285). (Respond: O. Oner, Gazi Ethem Pasa Sokak, Atala Apt 6/1, Senesenevler, 34742 Istanbul, Turkey).

COMMENT. The lower right prefrontal and frontal cerebral blood flow in ADHD subjects correlates with previous reports of an abnormal right frontal development on functional imaging studies (Castellanos FX et al. 1996; Ped Neur Briefs Aug 1996). Decreased volume of prefrontal cortex, caudate nucleus, and globus pallidus on the right side point to a dysfunction of the prefrontal-striatal circuits in ADHD.

\section{AGGRESSIVE BITING BEHAVIOR AND SEIZURES}

The biting behavior (BB) and other aggressive gestures in a group of 11 patients ( 8 male; 3 female) with drug-resistant seizures were studied in presurgical evaluations using video-EEG/SEEG monitoring at University of Bologna; Niguarda Hospital, Milan; and University of Modena, Italy. Seizure onset was at age 1 to 21 , and age at evaluation was 3059 years. BB was reflexive, occurring during or after a seizure, and evoked by intrusion of the examiner's hand near the patient's face. The act was associated with emotional arousal, fear, or anger, and was accompanied by aggressive gestures. The EEG showed that BB was localized to ictal involvement of the amygdala/hippocampal and the orbitomedial prefrontal regions. (Tassinari CA, Tassi L, Calandra-Buonaura $\mathrm{G}$ et al. Biting behavior, aggression, and seizures. Epilepsia May 2005;46:654-663). (Reprints: Dr S Meletti, Division of Neurology, Bellaria Hospital, University of Bologna, Via Altura 3, Bologna 40139, Italy).

COMMENT. This study concerned adults whose seizures began in childhood. The authors emphasize that violence is exceptional during epileptic seizures, and distinct from that reported with postictal psychosis. The diagnosis requires EEG confirmation during BB. In children with ADHD and comorbid oppositional defiance disorder, biting and aggressive tendencies are not uncommon, and abnormal EEGs have been reported with ADHD in 15\% of cases (Hemmer SA, Pasternak JK et al, 2001; Ped Neur Briefs May 2003). A video-EEG is necessary to relate $\mathrm{BB}$ to ictal activity, as a prelude to a trial of antiepileptic medication. Several studies have linked behavioral abnormalities to epilepsy in childhood, but the indications for antiepileptic treatment are controversial (Boone KB et al, 1988; [Ped Neur Briefs April 1988]; Austin JK et al, 1992; [Ped Neur Briefs Jan 1993]; Wakai S et al, 1994 [Ped Neur Briefs Nov 1994]; Amir N et al, 1994 [Ped Neur Briefs Dec 1994]). 